\title{
Coexpression of Ang1 and Tie2 in Odontoblasts of Mouse Developing and Mature Teeth-A New Insight into Dentinogenesis
}

\author{
Kazunori Nakajima $^{1}$, Yasuaki Shibata ${ }^{2}$, Yoshitaka Hishikawa ${ }^{3,7}$, Takashi Suematsu ${ }^{4}$, \\ Masako Mori ${ }^{5}$, Shigetomo Fukuhara ${ }^{6}$, Takehiko Koji ${ }^{3}$, Takashi Sawase ${ }^{1}$ and \\ Tohru Ikeda ${ }^{2}$ \\ ${ }^{1}$ Department of Applied Prosthodontics, Nagasaki University Graduate School of Biomedical Sciences, ${ }^{2}$ Department of Oral \\ Pathology and Bone Metabolism, Nagasaki University Graduate School of Biomedical Sciences, ${ }^{5}$ Department of Cell Biology, \\ Nagasaki University Graduate School of Biomedical Sciences, 1-7-1 Sakamoto, Nagasaki 852-8588, Japan, ${ }^{3}$ Department of \\ Histology and Cell Biology, Nagasaki University Graduate School of Biomedical Sciences, ${ }^{4}$ Central Electron Microscope \\ Laboratory, Nagasaki University Graduate School of Biomedical Sciences, 1-12-4 Sakamoto, Nagasaki 852-8523, Japan, \\ ${ }^{6}$ Department of Cell Biology, National Cerebral and Cardiovascular Center Research Institute, 5-7-1 Fujishirodai, Suita, \\ Osaka 565-8565, Japan and 'Present address: Division of Histochemistry and Cell Biology, Department of Anatomy, \\ Faculty of Medicine, University of Miyazaki, 5200 Kihara, Kiyotake, Miyazaki 889-1692, Japan
}

Received November 25, 2013; accepted January 27, 2014; published online February 22, 2014

\begin{abstract}
Angiopoietin-1 regulates vascular angiogenesis and stabilization, and is reported to promote bone formation by facilitating angiogenesis. To estimate the role of Ang 1 in odontogenesis, we explored the distribution of Ang1 and the receptor, Tie2 in the mouse developing and mature first molar of the mandible. At embryonic day 18 , when differentiation of odontoblasts begins, immunosignals for Ang1 were intensely detected in the basement membrane and the distal side, which faced the basement membrane of odontoblasts. In situ hybridization revealed that Ang1 was expressed in odontoblasts and ameloblasts facing the basement membrane. Tie2 was localized in the distal side of odontoblasts. After birth. Ang1 was detected in the predentin, whereas both Ang1 and Tie2 were colocalized in odontoblasts and odontoblast processes. These distributions were retained up to 8 weeks. In contrast to odontoblasts, ameloblasts, cementoblasts and osteoblasts expressed Ang1 but did not express Tie2. Colocalization of Ang1 and Tie2 in odontoblasts and selective expression of Tie2 in odontoblasts among cells responsible for calcified tissue formation suggested the involvement of autocrine signals of Ang1-Tie2 in dentinogenesis.
\end{abstract}

Key words: angiopoietin-1, Tie2, odontoblast, dentinogenesis, tooth

\section{Introduction}

Odontoblasts are post-mitotic, neural crest-derived cells, which differentiate according to tooth-specific temporospatial patterns. Their terminal differentiation is characterized by several steps implying the withdrawal from the cell cycle, elongation, and cytological polarization, as well as transcriptional and translational modifications, that allow

Correspondence to: Yasuaki Shibata, Department of Oral Pathology and Bone Metabolism, Nagasaki University Graduate School of Biomedical Sciences, 1-7-1 Sakamoto, Nagasaki 852-8588, Japan. E-mail: siva@nagasaki-u.ac.jp the cells to synthesize the pre-dentin and dentin matrix [19]. It is now well known that the inner dental epithelium exerts control over the terminal differentiation of odontoblasts through basement membrane-mediated interactions. The basement membrane may act as a specific substrate and a reservoir for paracrine and autocrine factors [4]. Indeed, several growth factors, including the FGF family, the TGF $\beta$ superfamily and IGF1, which promote odontoblast differentiation, have been detected in the basement membrane [3, $4,11,19]$. These growth factors have also been shown to promote osteoblast growth, differentiation, and production of extracellular matrices $[12,14,16]$. As the precise mechanism of dentinogenesis is still not well defined, we con- 
jectured that certain factors that associate with the basement membrane and their receptors expressed in odontoblasts among cells responsible for calcified tissue formation might be involved in dentin-specific morphogenesis and function.

Angiopoietin-1 (Ang1) plays essential roles in balancing angiogenesis and vascular stabilization $[2,6]$. Tie2 is a receptor phosphotyrosine kinase that is preferentially expressed in the vascular endothelium. Tie 2 is a member of the Tie family and functions as a receptor for Ang1 $[2,6]$. The Ang1 family consists of Ang1, Ang2, Ang3, and Ang4. Of these, Ang1 and Ang2 are well characterized. Ang1, predominantly expressed in perivascular cells such as pericytes, fibroblasts, and tumor cells, binds to and activates Tie2 in a paracrine manner, while Ang2, which has similar affinity to Tie2, competes with Ang1 to bind with Tie2. Ang1-Tie2 is essential for ischemia-induced angiogenesis and tumor angiogenesis $[2,6]$. Mice that are deficient in either Ang1 or Tie2 have disrupted vasculature, resulting in increased permeability, compromised vascular function and ultimately embryonic death [5, 23]. In vitro experiments showed that Ang1 has the ability to bind with components of the basement membrane as well as to type I collagen [7, 29]. Moreover, several reports described that osteoblasts expressed Ang1 but not Tie2 [24, 28, 31], and that Ang1 induced bone formation by facilitating angiogenesis [24], whereas others reported that Ang1 synergistically enhanced BMP-induced bone formation [10]. However, the expression of Ang1 and Tie2 in tooth has yet to be examined.

In this study, we investigated the expression and localization of Ang1 and Tie2 in the developing and mature teeth to estimate the role of Ang1 in odontogenesis.

\section{Materials and Methods}

\section{Tissue preparation}

All experiments were reviewed by the Committee on the Guidelines for Animal Experimentation of Nagasaki University and performed according to the recommendations or under the conditions proposed by the committee. Pregnant and 8-week-old ICR mice were obtained from Texam Corp. (Nagasaki, Japan). At embryonic day 17 (E17), E18 postnatal day 3 (P3), P8 and P15, the animals were sacrificed under deep anesthesia, and the mandibles were excised and then immersed in $4 \%$ paraformaldehyde (PFA) in $0.01 \mathrm{M}$ phosphate-buffered saline (PBS, pH 7.4) at $4{ }^{\circ} \mathrm{C}$ overnight. Following decalcification with $10 \%$ ethylenediamine-N,N,N',N'-tetraacetic acid (EDTA) for 3 days at $4^{\circ} \mathrm{C}$, the specimens were dehydrated, embedded in paraffin and sagittally sectioned at a thickness of $4 \mu \mathrm{m}$. Eight-week-old mice were sacrificed, the mandibles were resected and fixed as described above, and then decalcified for 2 weeks with $10 \%$ EDTA. Some sections were stained with hematoxylin and eosin (HE), and examined under a light microscope. For western blotting, tissue lysate were prepared from first molar tooth germs of the mandible of ICR mice at E18. The excised tooth germs were homogenized in the presence of $50 \mu$ l of RIPA lysis buffer (Atto
Corp., Tokyo, Japan) with Biomasher (Nippi, Inc., Tokyo, Japan) on ice, and centrifuged at $15,000 \mathrm{rpm}$ at $4{ }^{\circ} \mathrm{C}$. The supernatant was used as loading samples.

\section{Immunohistochemical and immunofluorescent microscopic analyses}

Immunohistochemistry was performed as reported previously [18]. Sections were immersed in $0.3 \%$ hydrogen peroxidase to block endogenous peroxidase activity. After incubation with $1 \%$ bovine serum albumin (BSA) in PBS for $30 \mathrm{~min}$ at room temperature (RT), the specimens were reacted with antibodies for Ang1 diluted to 1:200, Tie2 diluted to 1:50 (both from Santa Cruz Biotechnology Inc., Santa Cruz, CA, USA) or nestin diluted to 1:200 (EMD Millipore Corp., Billerica, MA, USA) for $1 \mathrm{hr}$ at RT. As secondary antibodies, horseradish peroxidase (HRP)conjugated anti-rabbit or anti-mouse antibodies (Amersham Biosciences Corp., Piscataway, NJ, USA) were used. Immunosignals were visualized with 3,3'-diaminobenzidine (Sigma Aldrich, Steinheim, Germany). To retrieve antigens for the anti-Angl antibody, specimens were treated with $1 \mu \mathrm{g} / \mathrm{ml}$ proteinase $\mathrm{K}$ (Wako Pure Chemical Industries, Osaka, Japan) for $10 \mathrm{~min}$ at RT before the blocking procedure. Sections were counterstained with methyl green. For immunofluorescent microscopic analysis, Alexa Fluor 488 goat anti-rabbit antibody diluted to 1:400 and Alexa Fluor 594 rabbit-anti goat antibody diluted to 1:400 were used as secondary antibodies (both from Molecular Probes, Eugene, OR, USA). For double immunofluorescent microscopic analysis of Ang1 and nestin, antigen retrieved with proteinase $\mathrm{K}$ was omitted to protect immunosignals for nestin. 4',6-diamidino-2-phenylindole (DAPI) was used for counterstaining. Fluorescent signals were visualized using a confocal laser microscope (LSM5 PASCAL; Carl Zeiss, Oberkochen, Germany).

\section{In situ hybridization}

DNA fragments of mouse Ang1 were amplified using mouse kidney cDNA as a template with 5'primer (5'-AAGAGCAAGCTTTGCAGGAG-3') and 3'primer (5'CAAGTTTTTGCAGCCACTGA-3'), and subcloned into pGEM-T easy vector. Digoxigenin-labeled complementary RNA probes were produced with T7 polymerase for antisense and Sp6 polymerase for sense (both from Life Technologies, Carlsbad, CA, USA). In situ hybridization was performed as described previously, with slight modification [21]. After treatment with $10 \mu \mathrm{g} / \mathrm{ml}$ proteinase $\mathrm{K}$ (Wako Pure Chemical Industries) for $15 \mathrm{~min}$ at $37^{\circ} \mathrm{C}$, sections were hybridized with probes $(1 \mu \mathrm{g} / \mathrm{ml})$ for $16 \mathrm{hr}$ at $37^{\circ} \mathrm{C}$. After washing with $0.1 \times \mathrm{SSC}$ at $40^{\circ} \mathrm{C}$, sections were treated with $0.5 \mu \mathrm{g} / \mathrm{ml}$ ribonuclease A (Wako Pure Chemical Industries) for $30 \mathrm{~min}$ at $37^{\circ} \mathrm{C}$. The signals were visualized using alkaline phosphatase-conjugated anti-digoxigenin antibody Fab fragments with 4-nitro blue tetrazolium salt/5-bromo4-chloro-3-indolyl-phosphate (both from Roche Diagnostics, Mannheim, Germany). 


\section{Western blotting}

Lysate of tooth germ at E18 (30 $\mu \mathrm{g} / \mathrm{lane})$ was separated under reducing conditions on 5\% SDS polyacrylamide gel (Super Sep, Wako Pure Chemical Industries, Osaka, Japan) and transferred to PVDF membrane (Atto Corp., Tokyo, Japan). The blot was blocked with 5\% BSA in Tris-buffered saline with $0.2 \%$ tween 20 (TBST) overnight at $4{ }^{\circ} \mathrm{C}$ and incubated with anti-Tie 2 or anti-phosphorylated Tie 2 antibodies (1:1000, Merck Millipore, Darmstadt, Germany) for $3 \mathrm{hr}$ at RT. After washing with TBST, HRP-conjugated anti-rabbit or anti-mouse antibodies (1:2000, Amersham Biosciences Corp., Piscataway, NJ, USA) were applied and incubated for $1 \mathrm{hr}$ at RT. Following the final wash, the blot was incubated with ECL Prime Western Blotting Detection Reagent and the signal was detected with image analyzer, ImageQuant LAS 4000mini (both from GE Healthcare UK Ltd., Buckinghamshire, UK).

\section{Results}

\section{Distribution of Ang1 and Tie2 at the onset of odontoblast differentiation}

In the first molar of mouse mandible, differentiation of odontoblasts begins in mesenchymal cells of the dental papilla at the tip of the future cusp at E18 (Fig. 1a). For the purpose of illustration, the differentiation sequence of odontoblasts and their positional relationships to ameloblasts are shown in Figure 1b. Mesenchymal cells in contact with the basement membrane differentiate into odontoblasts. Mature odontoblasts are columnar polarized cells with eccentric nuclei, odontoblast processes, and secretion of dentin matrices. After deposition of dentin matrices, preameloblasts opposing odontoblasts fully differentiate and begin to produce enamel matrix.

We explored the distribution of Ang 1 and Tie2 at the onset of odontoblast differentiation in the first molar of the mouse mandible at E18. Immunosignals for anti-Ang1 antibody were intensely detected around the region of basement membrane of the crown except for the cervical loop (Fig. 1c). Higher magnification showed that Ang1immunosignals were localized at the distal side of odontoblasts and basement membrane, and faintly in ameloblasts (Fig. 1c, d, e). At the site near the occlusal pit, preameloblasts were negative for Ang1 (Fig. 1c, d). In situ hybridization for Ang1 mRNA also showed that Ang1 was expressed in odontoblasts and ameloblasts, but not in preameloblasts (Fig. 1f). Immunosignals for anti-Tie2 antibody were localized in the distal side of odontoblasts (Fig. 1g, h). Cellular processes of columnar odontoblasts elongating into dentin matrices were positively stained with Tie2 antibody (Fig. 1g). Endothelial cells of the capillaries in the pulp horn were also positive for Tie2 (Fig. 1h). On the contrary, mesenchymal cells adjacent to epithelium at E17, one day before the beginning of odontoblast differentiation, were Tie2-negative, although endothelial cells at outside of the tooth germ were Tie2-positive (Fig. 1i). Western blotting was performed to confirm the expression of Tie2 in tooth germ of mandible at E18. As shown Figure 1i, anti-Tie2 antibody labeled two bands at approximately $150 \mathrm{kDa}$. Immunoblotting with anti-phosphorylated Tie 2 antibody labeled a single band, estimated to the lower band reacted with anti-Tie 2 antibody.

\section{Distribution of Ang1 and Tie2 in the developing tooth after birth}

After birth, the layer of odontoblasts elongates to form the root of the tooth. Unlike osteocytes, odontoblasts are never entrapped in the dentin matrix, but the cell process that is left behind in the dentin matrix constitutes the odontoblast process surrounded by the dentinal tubule. We performed immunohistochemical analyses for Ang1 and Tie 2 proteins in the mouse mandible at P3, P8 and P15. At $\mathrm{P} 3$, immunosignals for anti-Ang1 and anti-Tie2 antibodies were extended in the whole crown except for the cervical loop (Fig. 2a, d, e). Ang1 immunosignals were detected in the predentin and odontoblasts (Fig. 2a), whereas Tie2 immunosignals were strictly localized at the distal side of odontoblasts including odontoblast processes (Fig. 2d, e). Ameloblasts were positive for Ang1 (Fig. 2a, b) but negative for Tie2 after birth (Fig. 2d, e). At P8, when crown formation was almost completed, Ang1 immunosignals in odontoblasts were decreased compared with those at P3, although those in predentin remained (Fig. 2b). In the developing root at P15, immunosignals for anti-Ang1 antibody were localized in odontoblasts and the dentin matrix, whereas Tie 2 was localized in the distal side of odontoblasts including odontoblast processes, just like in the crown (Fig. 2c, f). Osteoblasts, cementoblasts and cells in periodontal ligament were also positive for Ang1, but negative for Tie2 (Fig. 2c). Fibers in periodontal ligament were also positive for Ang1 (Fig. 2c).

\section{Confocal laser microscopic findings of immunosignals for Ang1 and Tie2}

To determine the exact odontoblast stage that produces each protein, we performed double immunofluorescent microscopic analysis by using antibody against nestin, an intermediate filament that has been used as a marker of odontoblasts [9, 26]. At E18, immunosignals for Ang1 were detected in a subset of nestin-positive odontoblasts (Fig. 3a). The basement membrane was not stained with Angl-antibody because the antigen retrieval step with proteinase $\mathrm{K}$ was omitted to protect the immunosignals for nestin. At 8 weeks after birth, immunosignals of Ang1 and Tie2 were colocalized at the distal side of odontoblasts. Ang1 signals were also seen in the predentin (Fig. 3c). At higher magnification, the colocalization of Ang 1 and Tie2 in dentin tubuli was clearly seen (Fig. 3d).

\section{Discussion}

Ang1 is known to be a potent angiogenic inducer, but little is known regarding its effect on non-endothelial cells. In this study, we showed Ang1 was expressed in odonto- 
a

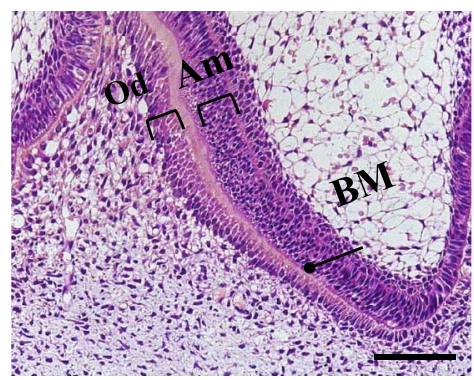

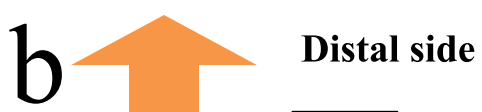

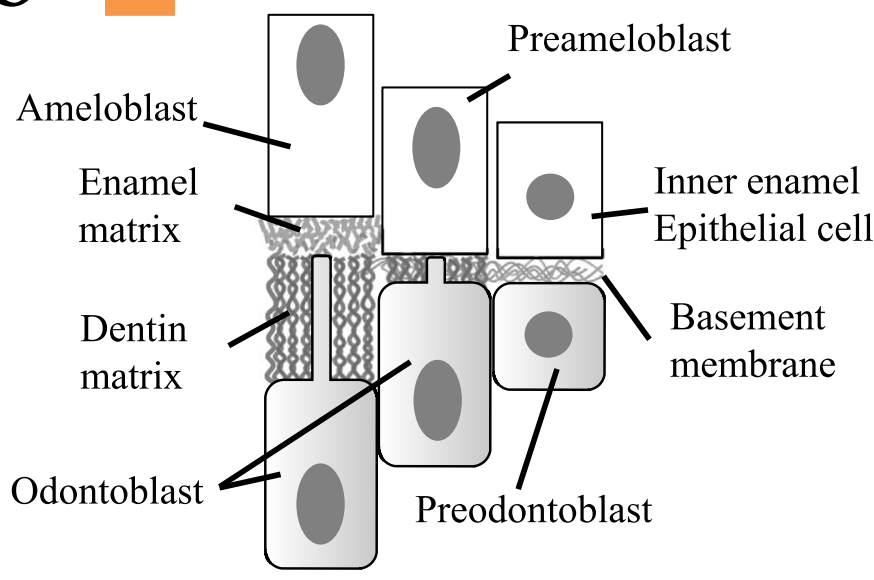

Proximal side
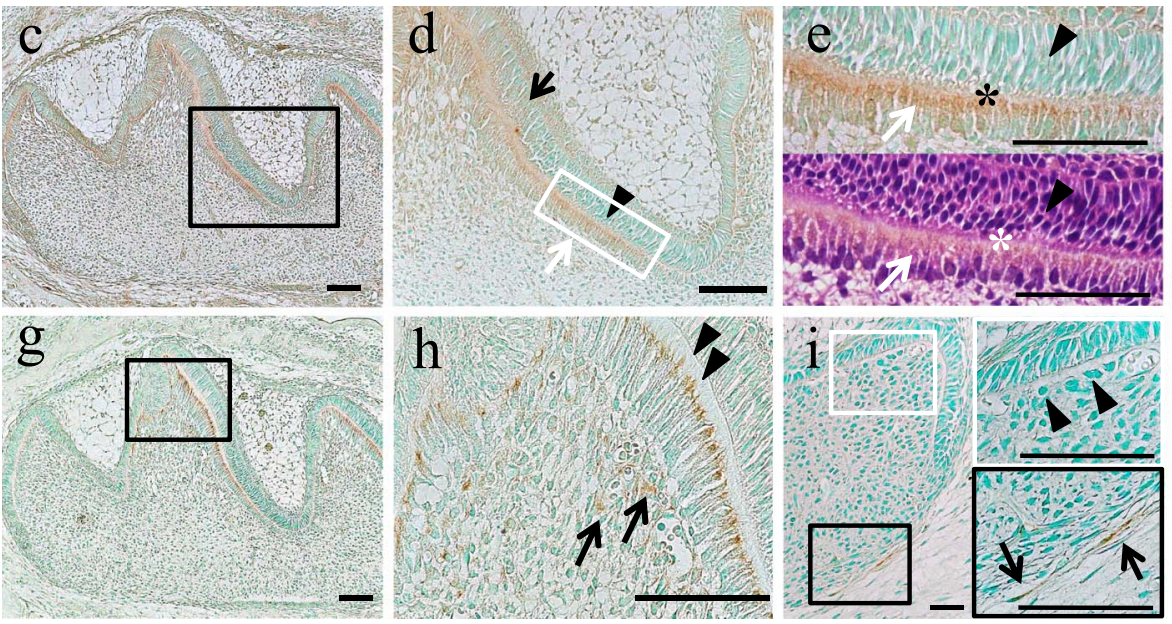

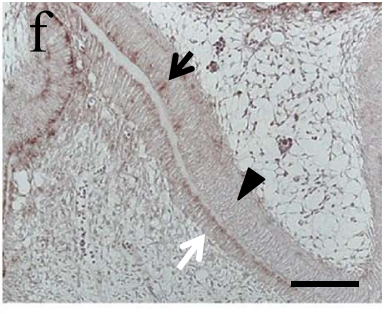

j

Tie2 pTie2

$150 \mathrm{kDa}$

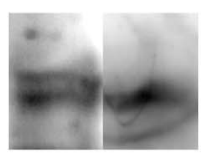

Fig. 1. Distribution of Ang1 and Tie2 in the first molar tooth of the mouse mandible at E17 and E18. (a) HE staining of the first molar of mandible at E18. (b) A schema of odontoblast differentiation, showing their positional relationships with basement membrane and ameloblasts. (c, d, e) Immunohistochemistry for Ang1 in the first molar at E18. (d) Higher magnification of the rectangle in (c). (e) Higher magnification of the rectangle in (d). Lower panel is HE staining of the region corresponded to upper one. Ang1 immunosignals in the distal side of odontoblasts (white arrows) and the proximal side of ameloblasts (a black arrow) in addition to the basement membrane (asterisks). Preameloblasts (an arrowhead) are negative for Ang1. (f) In situ hybridization for Ang1 mRNA. Positive signals in odontoblasts (a white arrow) and ameloblasts (a black arrow) and negative signals in pre-ameloblasts (an arrowhead). (g) Tie2 immunosignals in the central and distal cusps. (h) Higher magnification of the rectangle in (g). Cellular processes of odontoblasts (arrowheads) are stained positively. Endothelial cells in the pulp horn circumscribing capillaries are Tie2-positive (black arrows). (i) Immunostaining for Tie2 of the first molar tooth germ of mandible at E17 as a control. White and black rectangles are magnified at right panels. Undifferentiated mesenchymal cells (arrowheads) are Tie2-negative, but endothelial cells are Tie2-positive (arrows). (i) Western blotting of first molar tooth germ of mandible at E18 with anti-Tie2 antibody (left) and anti-phosphorylated Tie2 antibody. All bars $=80 \mu \mathrm{m}$.

blasts and deposited in the basement membrane, and subsequently in the dentin matrix and predentin. Double immunofluorescent microscopic analysis with anti-nestin antibody confirmed that nestin-positive differentiated odontoblast expressed Ang1 and Tie2. Western blotting revealed expression of Tie 2 and its phosphorylation in tooth germ at E18. These results suggested that Ang1 and Tie2 were coexpressed in differentiated odontoblasts in developing and mature tooth and generated autocrine signals.

Immunosignals for Tie 2 in osteoblasts were negligible in contrast to those in odontoblasts, as described previously $[24,28,31]$. In addition, we found that cementoblasts and 

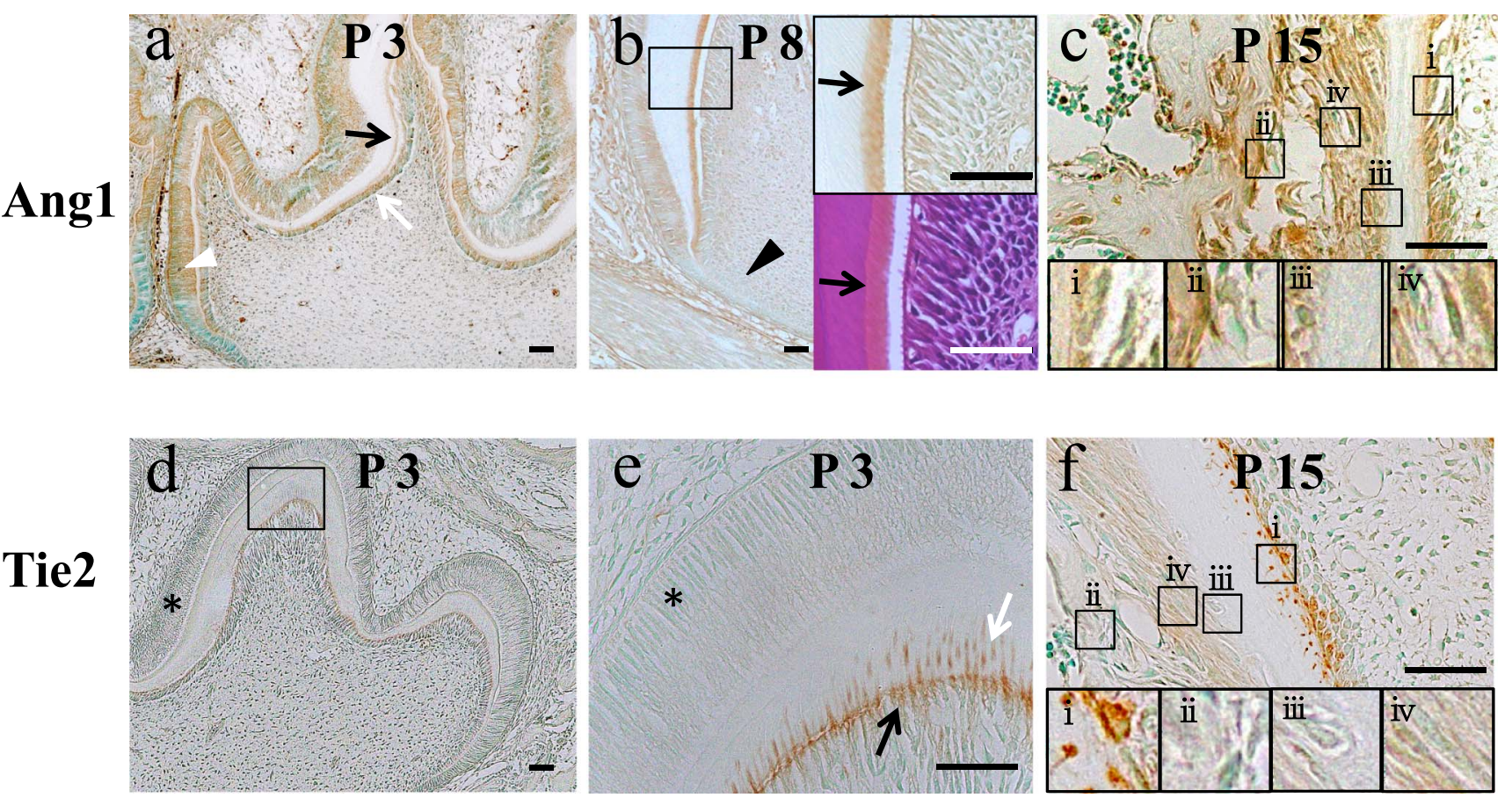

Fig. 2. Distribution of Ang1 and Tie2 after birth. (a) Ang1 immunosignals in odontoblasts (a white arrow), predentin (a black arrow), ameloblasts (a white arrowhead) at P3. (b) Ang1 immunosignals at P8. Right upper panel is higher magnification of the rectangle in left panel. Right lower panel is HE staining of the same field with upper one. Predentin is clearly stained with Ang1-antibody. Hertwig's epithelial root sheath is Ang1-negative (a black arrowhead). Ang1 immunosignals in the root of the developing tooth at P15. Odontoblasts (i), osteoblasts (ii), cementoblasts (iii) and periodontal ligament (iv) are Ang1-positive. (d) Tie2 immunosignals at P3. (e) Higher magnification of the rectangle in (d). Tie2 signals are detected in odontoblast processes (a white arrow) embedded in the dentin matrix as well as the distal side of odontoblasts (a black arrow). (f) In the mandible at P15, Tie2 signals are detected in odontoblasts (i) but not in osteoblasts (ii), cementoblasts (iii), and periodontal ligament (iv). Note that ameloblasts (asterisks in d, e) are also Tie2-negative. Bar=80 $\mu \mathrm{m}$.

ameloblasts were negative for immunosignals for Tie2. These results indicated selective expression of Tie2 in odontoblasts among cells participating in calcified tissue formation, and suggested the involvement of Ang1-Tie2 signals in dentin-specific morphology and function. Some reports suggested that the autocrine signaling of Ang1 is involved in several pathological conditions, such as rheumatoid arthritis [25], gastric cancer [27], thyroid tumors, and adenomatous goiters [17]. To the best of our knowledge, however, there are no reports of autocrine signaling of Ang1-Tie2 under physiological conditions. This is the first observation of the possible involvement of autocrine Tie2 signaling in physiological cells.

Ang1 has the ability to bind with type I collagen, and the components of the basement membrane [7, 29]. As type I collagen, the main component of dentin matrices, is deposited into the basement membrane at an early stage of dentinogenesis [19]. We conjectured that Ang1 in the basement membrane might contribute to promote the deposition of dentin matrices in the basement membrane. In addition, two groups demonstrated that Tie2 was recruited and anchored to cell-substratum contacts by extracellular matrixbound Ang1 to form novel adhesive structures, which are different from focal contacts that generally involve integrins
$[7,20]$. On the adhesive structures, the phosphorylated Tie2 activates Erk either through accelerating the formation of focal contacts or partly activating FAK, a component of focal contact, or both, and consequently induces the migration of endothelial cells. Migration entails the polarization of the cell, and subsequent changes of cell shape are mostly driven by the reorganization of the cytoskeleton [22]. In addition, the expression of integrin ev $\beta 3$ in both odontoblasts and endothelial cells has been reported [13]. Our results suggested that Ang1 deposited in the basement membrane recruited Tie2 to the distal side, and that the signal regulated cellular dynamics by promoting focal contact and reconstruction of the cytoskeleton, which organized the odontoblast-specific morphology in the initial stage of dentinogenesis. In addition, the adhesive structures formed by Ang1 and Tie2 in mature dentin would seem to play roles in the homeostasis of the odontoblasts.

Previous electron microscopic study revealed that capillaries began to invade into the odontoblast layer during odontogenesis and were finally located close to the predentin in accordance with the active production of dentin matrices by odontoblasts [30]. The expression of Ang1 in odontoblasts and Tie2 in endothelial cells in the pulp horn at the onset of odontoblast differentiation suggested that 

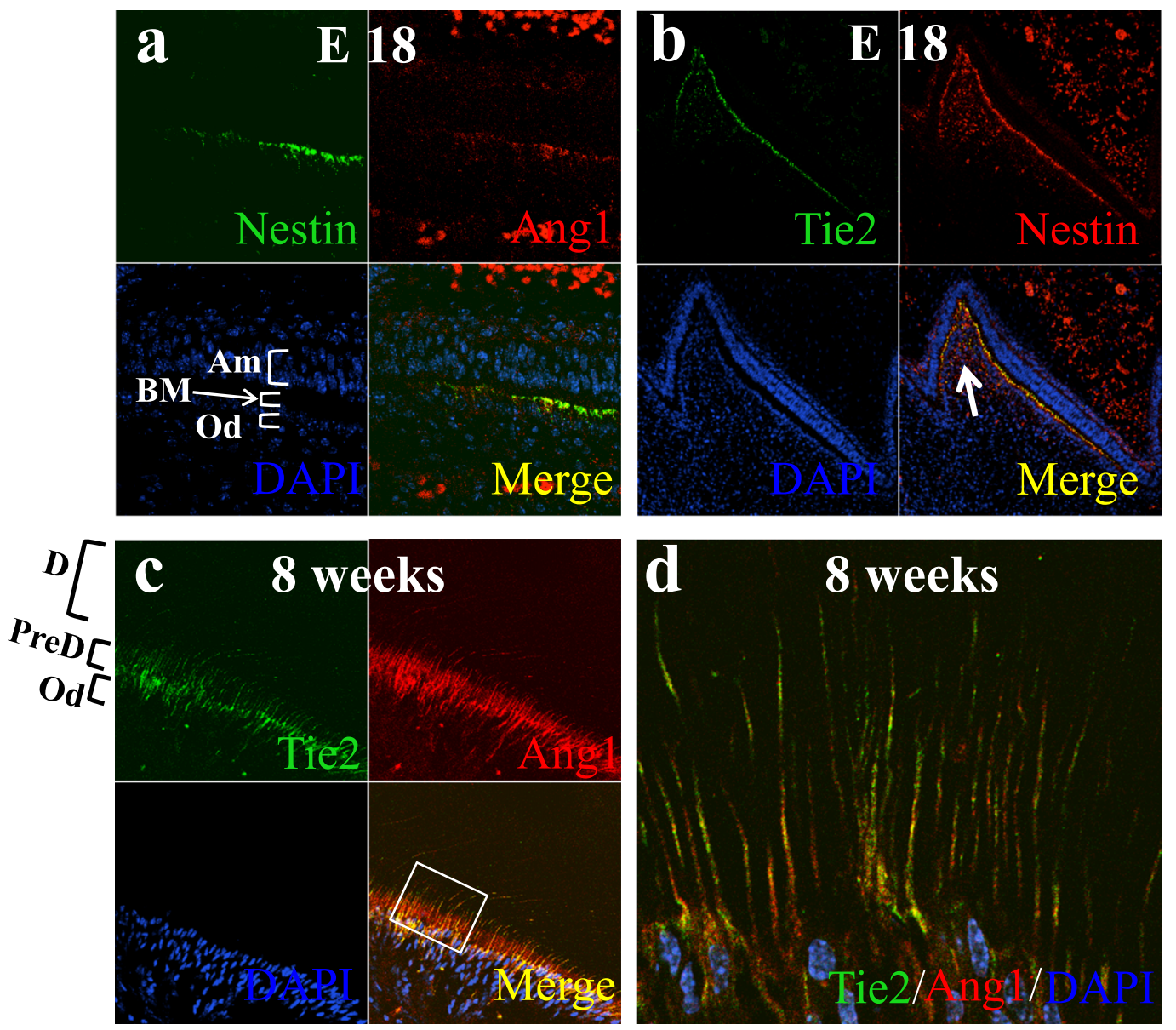

Fig. 3. Double immunofluorescent confocal laser microscopic analysis. (a) Distribution of Ang1 (red) and nestin (green) in differentiating odontoblasts $(\mathrm{Od})$ in contact with the basement membrane (BM), facing pre-ameloblasts (Pre Am) at E18. Ang1 is localized in nestin-positive odontoblast. Without antigen retrieved with proteinase $\mathrm{K}$ to protect immunosignals for nestin, Ang1 immunosignals in the basement membrane were undetectable. Original magnification, $\times 60$. (b) Both Tie2 (green) and nestin (red) are detected in odontoblasts and cells in the pulp horn (a white arrow) at E18. Original magnification, $\times 20$. (c) Colocalization of Tie2 (green) and Ang1 (red) in the first molar of the mandible at 8 weeks after birth. Od, a layer of odontoblasts; PreD, predentin; D, dentin; original magnification, $\times 60$. (d) Colocalization of Tie2 (green) and Ang1 (red) in odontoblast processes in the first molar of the mandible at 8 weeks after birth. Original magnification, $\times 100$.

Ang1 secreted from odontoblasts contributed to the invasion of capillaries into the odontoblast layer.

The expression of Ang1 in ameloblasts and the deposition of Ang1 in the basement membrane, but not in the enamel matrix, suggested that ameloblasts also contributed to the deposition of Ang1 in the basement membrane, but Ang1 was not directly involved in amelogenesis. Interestingly, previous studies showed the expression of vascular endothelial growth factor in ameloblasts $[1,8]$. We conjectured that the expression of Ang1 in ameloblasts may contribute to induce capillaries in the enamel organ. Nestin is known to be one of the markers not only of odontoblasts $[9,26]$, but also of endothelial cells of immature and newly formed microvessels [15]. These results suggested an association of the biological mechanisms of odontogenesis with those of angiogenesis.

In conclusion, we showed that Angl was deposited in the basement membrane and dentin matrix, and its receptor Tie2 was selectively expressed in odontoblasts among the cells responsible for calcified tissue formation. Colocalization of Ang1 and Tie2 in odontoblasts from embryonic to adult stages suggested their involvement in dentinogenesis and the homeostasis of odontoblasts. In addition, Tie 2 could be a new useful marker to identify odontoblasts among other cells responsible for calcified tissue formation. To elucidate the biological significance of Ang1-Tie2 complex in dentinogenesis, further functional analysis is required.

\section{Acknowledgments}

This study was funded by Nagasaki University. The authors declare no potential conflicts of interest with respect to the authorship and/or publication of this article. 


\section{References}

1. Aida, M., Irié, T., Aida, T. and Tachikawa, T. (2005) Expression of protein kinases $\mathrm{C} \beta \mathrm{I}, \beta \mathrm{II}$, and VEGF during the differentiation of enamel epithelium in tooth development. J. Dent. Res. 84; 234-239.

2. Brindle, N. P. J., Saharinen, P. and Alitalo, K. (2006) Signaling and functions of angiopoietin-1 in vascular protection. Circ. Res. 98; 1014-1023.

3. Cam, Y., Neumann, M. R., Oliver, L., Raulais, D., Janet, T. and Ruch, J. V. (1992) Immunolocalization of acidic and basic fibroblast growth factors during mouse odontogenesis. Int. J. Dev. Biol. 36; 381-389.

4. Catón, J. and Tucker, A. S. (2009) Current knowledge of tooth development: patterning and mineralization of the murine dentition. J. Anat. 214; 502-515.

5. Dumont, D. J., Gradwohl, G., Fong, G. H., Puri, M. C., Gertsenstein, M., Auerbach, A. and Breitman, M. L. (1994) Dominant-negative and targeted null mutations in the endothelial receptor tyrosine kinase, tek, reveal a critical role in vasculogenesis of the embryo. Genes Dev. 8; 1897-1909.

6. Fiedler, U. and Augustin, H. G. (2006) Angiopoietins: a link between angiogenesis and inflammation. Trends Immunol. 27; $552-558$

7. Fukuhara, S., Sako, K., Minami, T., Noda, K., Kim, H. Z., Kodama, T., Shibuya, M., Takakura, N., Koh, G. Y. and Mochizuki, N. (2008) Differential function of Tie2 at cell-cell contacts and cell-substratum contacts regulated by angiopoietin-1. Nat. Cell Biol. 10; 513-526.

8. Ide, S., Tokuyama, R., Davaadorj, P., Shimozuma, M., Kumasaka, S., Tatehara, S. and Satomura, K. (2011) Leptin and vascular endothelial growth factor regulate angiogenesis in tooth germs. Histochem. Cell Biol. 135; 281-292.

9. Imaizumi, Y., Amano, I., Tsuruga, E., Kojima, H. and Sawa, Y. (2010) Immunohistochemical examination for the distribution of podoplanin-expressing cells in developing mouse molar tooth germs. Acta Histochem. Cytochem. 43; 115-121.

10. Jeong, B. C., Kim, H. J., Bae, I. H., Lee, K. N., Lee, K. Y., Oh, W. M., Kim, S. H., Kang, I. C., Lee, S. E., Koh, G. Y., Kim, K. K. and Koh, J. T. (2010) COMP-Ang1, a chimeric form of Angiopoietin 1 , enhances BMP2-induced osteoblast differentiation and bone formation. Bone 46; 479-486

11. Joseph, B. K., Savage, N. W., Daley, T. J. and Young, W. G. (1996) In situ hybridization evidence for a paracrine/autocrine role for insulin-like growth factor-I in tooth development. Growth Factors 13; 11-17.

12. Linkhart, T. A., Mohan, S. and Baylink, D. J. (1996) Growth factors for bone growth and repair: IGF, TGF $\beta$ and BMP. Bone $19 ; 1 \mathrm{~S}-12 \mathrm{~S}$

13. Lucchini, M., Couble, M.-L., Romeas, A., Staquet, M.-J., Bleicher, F., Magloire, H. and Farges, J. C. (2004) $\alpha v \beta 3$ integrin expression in human odontoblasts and co-localization with osteoadherin. J. Dent. Res. 83; 552-556.

14. Marie, P. J., Miraoui, H. and Sévère, N. (2012) FGF/FGFR signaling in bone formation: progress and perspectives. Growth Factors 30; 117-123.

15. Matsuda, Y., Hagio, M. and Ishiwata, T. (2013) Nestin: a novel angiogenesis marker and possible target for tumor angiogenesis. World J. Gastroenterol. 19; 42-48.

16. Minuto, F., Palermo, C., Arvigo, M. and Barreca, A. M. (2005) The IGF system and bone. J. Endocrinol. Invest. 28; 8-10.

17. Mitsutake, N., Namba, H., Takahara, K., Ishigaki, K., Ishigaki, J.,
Ayabe, H. and Yamashita, S. (2002) Tie-2 and angiopoietin-1 expression in human thyroid tumors. Thyroid 12; 95-99.

18. Nakazawa, M., Obata, Y., Nishino, T., Abe, S., Nakazawa, Y., Abe, K., Furusu, A., Miyazaki, M., Koji, T. and Kohno, S. (2013) Involvement of leptin in the progression of experimentally induced peritoneal fibrosis in mice. Acta Histochem. Cytochem. 46; 75-84.

19. Ruch, J. V., Lesot, H. and Bègue-Kirn, C. (1995) Odontoblast differentiation. Int. J. Dev. Biol. 39; 51-68.

20. Saharinen, P., Eklund, L., Miettinen, J., Wirkkala, R., Anisimov, A., Winderlich, M., Nottebaum, A., Vestweber, D. and Deutsch, U. (2008) Angiopoietins assemble distinct Tie2 signalling complexes in endothelial cell-cell and cell-matrix contacts. Nat. Cell Biol. 10; 527-537.

21. Shibata, Y., Tsukazaki, T., Hirata, K., Xin, C. and Yamaguchi, A. (2004) Role of a new member of IGFBP superfamily, IGFBP$\mathrm{rP} 10$, in proliferation and differentiation of osteoblastic cells. Biochem. Biophys. Res. Commun. 325; 1194-1200.

22. Sonnenberg, A., Lecuit, T., Rottner, K. and Stradal, T. E. (2011) Actin dynamics and turnover in cell motility. Curr. Opin. Cell Biol. 23; 569-578.

23. Suri, C., Jones, P. F., Patan, S., Bartunkova, S., Maisonpierre, P. C., Davis, S., Sato, T. M. and Yancopoulos, G. D. (1996) Requisite role of angiopoietin-1, a ligand for the TIE2 receptor, during embryonic angiogenesis. Cell 87; 1171-1180.

24. Suzuki, T., Miyamoto, T., Fujita, N., Ninomiya, K., Iwasaki, R., Toyama, Y. and Suda, T. (2007) Osteoblast-specific angiopoietin 1 overexpression increases bone mass. Biochem. Biophys. Res. Commun. 362; 1019-1025.

25. Takahara, K., Iioka, T., Furukawa, K., Uchida, T., Nakashima, M., Tsukazaki, T. and Shindo, H. (2004) Autocrine/paracrine role of the angiopoietin- 1 and $-2 /$ Tie 2 system in cell proliferation and chemotaxis of cultured fibroblastic synoviocytes in rheumatoid arthritis. Hum. Pathol. 35; 150-158.

26. Terling, C., Rass, A., Mitsiadis, T. A., Fried, K., Lendahl, U. and Wroblewski, J. (1995) Expression of the intermediate filament nestin during rodent tooth development. Int. J. Dev. Biol. 39; 947-956.

27. Wang, J., Wu, K., Zhang, D., Tang, H., Xie, H., Hong, L., Pan, Y., Lan, M., Hu, S., Ning, X. and Fan, D. (2005) Expressions and clinical significances of angiopoietin-1, -2 and Tie2 in human gastric cancer. Biochem. Biophys. Res. Commun. 337; 386-393.

28. Winslow, M. M., Pan, M., Starbuck, M., Gallo, E. M., Deng, L., Karsenty, G. and Crabtree, G. R. (2006) Calcineurin/NFAT signaling in osteoblasts regulates bone mass. Dev. Cell 10; 771782.

29. Xu, Y. and Yu, Q. (2001) Angiopoietin-1, unlike angiopoietin-2, is incorporated into the extracellular matrix via its linker peptide region. J. Biol. Chem. 276; 34990-34998

30. Yoshida, S. and Ohshima, H. (1996) Distribution and organization of peripheral capillaries in dental pulp and their relationship to odontoblasts. Anat. Rec. 245; 313-326.

31. Zhang, Y., Singh, M. K., Degenhardt, K. R., Lu, M. M., Bennett, J., Yoshida, Y. and Epstein, J. A. (2009) Tie2Cre-mediated inactivation of plexinD1 results in congenital heart, vascular and skeletal defects. Dev. Biol. 325; 82-93.

This is an open access article distributed under the Creative Commons Attribution License, which permits unrestricted use, distribution, and reproduction in any medium, provided the original work is properly cited. 